\title{
Retardantes de chamas comerciais em quatro variedades de eucalipto
}

\author{
Commercial flame retardants in four \\ varieties of eucalyptus
}

\author{
Vanessa Dummer Marques ${ }^{1}$, Débora Duarte Ribes ${ }^{1}$,
} Vitória Correia Mota Canil ${ }^{2}$, Darci Alberto Gatto ${ }^{1}$

\author{
${ }^{1}$ Programa de Pós-graduação em Ciência e Engenharia de Materiais - UFPEL, Pelotas, RS, Brasil. \\ e-mail: engvanessadummer@gmail.com \\ deboraribes@hotmail.com \\ darcigatto@yahoo.com \\ ${ }^{2}$ Faculdade de Engenharia Industrial Madeireira, UFPEL, Pelotas, RS, Brasil. \\ vitoriacanil@gmail.com
}

\section{RESUMO:}

Neste trabalho objetivou-se verificar a capacidade de retardância de chamas de dois produtos comerciais aplicados em quatro espécies florestais (Eucalyptus saligna, Eucalyptus dunnii, Corymbia maculata e Eucalyptus grandis) avaliando a sua perda de massa ao longo do processo de combustão e o comportamento das amostras quando submetidas a elevadas temperaturas. Neste, foram utilizadas 9 amostras de cada espécie. Para a caracterização física das espécies (massa aparente $\left(\mathrm{g} / \mathrm{cm}^{3}\right)$ e porosidade $(\%)$ ), foram preparados 15 amostras de cada, totalizando 96 corpos de prova escolhidos aleatoriamente $(2,5 \times 2,5 \times 2,5 \mathrm{~cm})$. Todos foram estabilizados a $12 \%$ de umidade em câmara climatizada. Após, com o auxílio de um pincel, foram aplicadas três camadas dos produtos aqui testados sobre as amostras, e estas foram aplicadas em intervalos de 24 horas. A diluição dos produtos obedeceu às recomendações de seus fabricantes. Para o processo de combustão das amostras, utilizou-se um bico de Bunsen, onde as amostras foram submetidas a chama oxidante deste até ocorrer a perda de $80 \%$ de sua massa ao longo do tempo. Durante o processo de combustão, a cada 30 segundos, verificou-se a perda de massa que ocorreu na amostra neste intervalo de tempo. Assim, foi possível obter curvas que permitiram analisar e caracterizar a resistência térmica da madeira com base na perda de massa das diferentes espécies aqui estudadas. Ambos os produtos se mostraram eficazes no que diz respeito a capacidade retardante de perda de massa das amostras quando em elevadas temperaturas, mas o produto a base de nitrogenados e boratos se mostra mais eficiente em relação ao produto a base de resina sintética pigmentada com aditivos ignífugos (tinta ignífuga). Com relação à eficácia destes sobre as madeiras, os resultados variam de acordo com a espécie estudada e isso, possivelmente, devido a composição química de cada variedade.

Palavras-chave: Eucalyptus, combustão, perda de massa da madeira, retardância de chamas.

\section{ABSTRACT:}

The objective of this work was to verify the flame retardancy capacity of two commercial products applied in four forest species (Eucalyptus saligna, Eucalyptus dunnii, Corymbia maculata and Eucalyptus grandis) evaluating their loss of mass throughout the combustion process and the behavior of the when subjected to high temperatures. In this, 9 samples of each species were used. For the physical characterization of the species (apparent mass $\left(\mathrm{g} / \mathrm{cm}^{3}\right)$ and porosity $\left.(\%)\right), 15$ samples of each were prepared, totalizing 96 randomly selected test pieces $(2.5 \times 2.5 \times 2.5 \mathrm{~cm})$. All were stabilized at $12 \%$ humidity in a heated room. Then, with the aid of a brush, three layers of the products tested on the samples were applied, and these were applied at 24 hour intervals. The dilution of the products obeyed the recommendations of their manufacturers. For the combustion of the samples, a Bunsen nozzle was used, where the samples were subjected to the flame of oxidation until the loss of $80 \%$ of its mass over time. During the combustion process, every 30 seconds, the mass loss occurred in the sample in this time interval. Thus, it was possible to obtain curves that allowed to analyze and to characterize the thermal resistance of the wood based on the loss of mass of the different species studied here. Both products proved to be effective as regards the retarding ability of mass loss of 
samples when at high temperatures, but the product based on nitrogen and borates is more efficient compared to the product based on synthetic resin pigmented with flame retardant additives (flame retardant paint). Regarding the effectiveness of these on the woods, the results vary according to the species studied and this possibly due to the chemical composition of each variety.

Key words: Eucalyptus, combustion, loss of wood mass, flame retardancy.

\section{INTRODUÇÃO}

A madeira apresenta-se como um material favorável para a construção civil, devido ao seu baixo custo, por se tratar de um recurso renovável, e ainda, por possuir excelentes propriedades tecnológicas. FIGUEROA e MOARES [1] e QUIRINO et al. [2] argumentaram que a madeira é um polímero natural e heterogêneo "tanto do ponto de vista físico, químico, mecânico e anatômico" devido a diferentes tipos de células e tecidos que ela apresenta e também a fatores relacionados a variações na disposição destas células e tecidos no sentido tangencial, radial e longitudinal da árvore. TRUGILHO et al. [3] afirmam que fatores genéticos, ambientais e também relacionados à idade da árvore, agem diretamente em suas características tecnológicas, possibilitando seu emprego para diversas finalidades.

Contudo, esse material lignocelulósico apresenta restrições relacionadas ao seu uso na construção civil, devido à sua susceptibilidade a incêndios [1], [4] e [5]. CASTRO [5] MORESCHI [6] e AGUILLAR FILHO [7] relataram que a espécie, densidade e componentes químicos da madeira são alguns dos fatores que influenciam na capacidade ou tendência da madeira para iniciar sua combustão. Além desses, outros fatores devem ser levados em consideração como potencializadores para a combustão da madeira, bem como: teor de umidade do material, temperatura da fonte de calor, tempo de exposição da madeira ao fogo, dimensões da peça e ainda, detalhes construtivos. Segundo RIBEIRO et al. [8], uma vez que o material é inflamado, um fator que influencia no risco é o quão rápido as chamas irão se propagar a partir do ponto de origem. $\mathrm{Na}$ madeira, esse risco pode ser reduzido ao mínimo e para tal, pode se fazer uso de retardantes de chamas impregnados ainda no processo de tratamento desta ou protegendo-se a superfície ou massa do material por meio de aplicação de tintas ou resinas intumescentes na região [9], visando preservar as suas características estéticas.

WILKIE e MORGAN [10] afirmaram que compostos retardantes à chama são substâncias utilizadas para tornar a propagação da chama mais lenta após iniciado seu processo de combustão, ou ainda, são utilizados para retardar o momento inicial de combustão do material, aumentando o tempo que a pirólise leva para iniciar. Dependendo da sua natureza, os retardantes de chamas podem possuir ação química ou física e atuar no estado sólido, liquido ou gasoso [11].

De acordo com PAULA et al. [11], os retardantes de chamas de ação física são produtos a base de magnésio, hidróxido de alumínio (alumina tri-hidratada) e alguns compostos de boro e fósforo, os quais atuam absorvendo grandes quantidades de calor do ambiente, evitando assim, a formação de gases tóxicos e inflamáveis durante a combustão, em consequência, evitam o processo de pirólise do material. Os retardantes de chamas de ação química são chamados de halógenos e não halógenos, os quais são constituídos de bromo, cloro e a base de fósforo, respectivamente. Estes não absorvem calor e consequentemente, o processo de pirólise ocorre liberando gases tóxicos e gerando fuligem.

Segundo ABARCA [12], um retardante à chama pode atuar através três maneiras no material, interferindo quimicamente no mecanismo de propagação da chama, produzindo gases que reduzam o suprimento de oxigênio $\left(\mathrm{O}_{2}\right)$, e assim, alimentando os gases necessários para que a chama ocorra, ou ainda, formando uma camada protetora que iniba a combustão do material.

Desse modo, neste trabalho objetivou-se verificar a capacidade de retardância de chamas de dois produtos comerciais aplicados em quatro espécies de madeira (Eucalyptus saligna, Eucalyptus dunnii, Corymbia maculata e Eucalyptus grandis), avaliando sua perda de massa ao longo do processo de combustão e o comportamento das amostras quando submetidas a elevadas temperaturas.

\section{MATERIAIS E MÉTODOS}

\subsection{Obtenção e preparo dos corpos de prova}

Para a realização deste estudo foram utilizadas amostras de madeiras de quatro espécies florestais (Eucalyptus grandis, Eucalyptus saligna, Corymbia maculata e Eucalyptus dunnii), com idades superiores a 20 anos, as quais foram disponibilizadas em toras de madeira pela Empresa Celulose Riograndense (CMPC), situada na cidade de Guaíba no Estado do Rio Grande do Sul (RS) no Brasil. Torna-se importante ressaltar 
que a escolha por madeiras do gênero Eucalyptus, ocorreu, pois, de acordo com a Revista da Madeira (Ed. $\mathrm{n}^{\mathrm{o}}$ 59, 2001), [13] tal gênero encontra-se em franca expansão no setor da construção civil.

Os retardantes de chamas comerciais utilizados são: líquido à base de compostos nitrogenados e boratos (CNB) e uma tinta ignífuga à base de resina sintética pigmentada com aditivos ignífugos (RSPAI). Além destes dois tratamentos, foram ensaiadas também as amostras sem tratamento, as quais serão aqui chamadas de amostras controle (CONT) A Tabela 1 descreve o preparo e o tratamento das amostras utilizadas neste estudo.

Tabela 1: Número de amostras ensaiadas e os tratamentos que cada espécie folhosa recebeu.

\begin{tabular}{l|l|l|l}
\hline \multicolumn{2}{|c}{} \\
\hline \multirow{4}{*}{ ESPÉCIES FLORESTAIS } & TRATAMENTOS & $\begin{array}{l}\text { DENSIDADE APARENTE (g// } \\
\text { cm }^{3} \text { E POROSIDADE (\%) }\end{array}$ & $\begin{array}{l}\text { ENSAIO DE COMBUSTÃO } \\
\text { (RETARDÂNCIA NA PERDA DE } \\
\text { MASSA) (\%) }\end{array}$ \\
\hline \multirow{4}{*}{ Eucalyptus grandis } & CNB & - & 3 \\
\cline { 2 - 4 } & RSPAI & - & 3 \\
\cline { 2 - 4 } & CONT. & 15 & 3 \\
\hline \multirow{5}{*}{ Corymbia maculata } & CNB & - & 3 \\
\cline { 2 - 4 } & RSPAI & - & 3 \\
\cline { 2 - 4 } & CONT. & 15 & 3 \\
\hline & CNB & - & 3 \\
\cline { 2 - 4 } & RSPAI & - & 3 \\
\cline { 2 - 4 } & CONT. & 15 & 3 \\
\hline & CNB & - & 3 \\
\cline { 2 - 4 } & RSPAI & - & 36 \\
\cline { 2 - 4 } & CONT. & 15 & \\
\hline
\end{tabular}

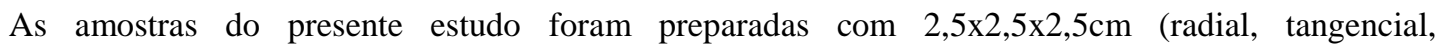
longitudinal) e escolhidas aleatoriamente entre as toras de madeira que foram doadas pela CMPC. É importante ressaltar que amostras com defeitos como: excesso de resinas e nós, foram descartadas.

Para obter a homogeneidade do teor de umidade (TU) das amostras em $12 \%$, de acordo com MORESCHI [6], após o corte, todas ficaram acondicionadas em uma câmara climatizada com temperatura e umidade do ar controladas em $20^{\circ} \mathrm{C}$ e $65 \%$ respectivamente, durante um período de quatro semanas.

Os retardantes de chamas usados foram aplicados em três camadas (demãos) sobre as amostras, conforme a Figura 1 (B), a qual apresenta as amostras tratadas com o retardante RSPAI e a Figura 1 (C) tratada com o retardante CNB. Já a Figura 1 (A) apresenta as amostras controle. É importante ressaltar que os produtos foram aplicados com o auxílio de um pincel e cada aplicação ocorreu em intervalo de 24 horas, respeitando as recomendações dos fabricantes quanto ao teor de diluição destes. Efetuados os tratamentos, as amostras foram acondicionadas novamente em câmara climatizada nas condições de equilíbrio já citadas, possibilitando assim à determinação de suas propriedades tecnológicas de interesse.
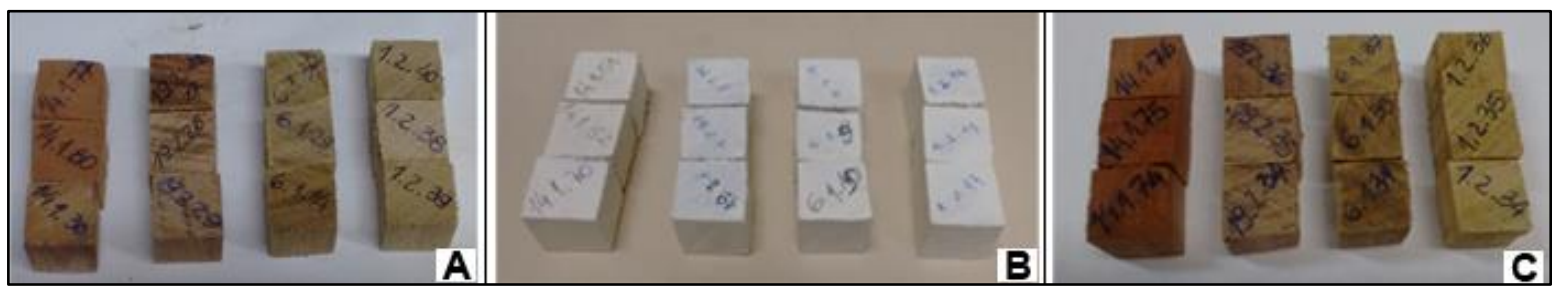

Figura 1: Amostras controle (sem tratamento) e tratadas destinadas ao ensaio de combustão. 


\section{1 - Propriedades físicas avaliadas}

Visando obter a densidade aparente (12\% TU) das espécies florestais (Equação 1), a massa e o volume destas foram verificadas utilizando-se uma balança de precisão e um paquímetro digital respectivamente.

A densidade básica e a porosidade foram determinadas por meio do emprego das Equações 2 e 3 , respectivamente.

$$
\rho_{12}=\frac{M_{12}}{V_{12}}
$$

Em que:

$\rho_{12}=$ densidade aparente $\left(\mathrm{g} / \mathrm{cm}^{3}\right)$;

$M_{12}=$ peso da amostra ou massa da amostra $(\mathrm{g}) ;$

$V_{12}=$ volume da amostra em condição de equilíbrio higroscópico $(12 \% \mathrm{TU})\left(\mathrm{cm}^{3}\right)$.

Determinou-se a densidade básica (Equação 2) conforme recomendações da Norma Brasileira regulamentadora - NBR 7190 da Associação Brasileira de Normas Técnicas - ABNT (1997), enquanto a porosidade foi calculada com base na metodologia descrita por MORESCHI [6] e Equação 3.

$$
\begin{aligned}
& \rho_{e b}=\frac{M_{0}}{V_{s}} \\
& \Phi=\left\lceil 1-\left(\frac{\rho_{e b}}{1,54}\right)\right] \times 100
\end{aligned}
$$

Em que:

$\rho_{e b}=$ densidade específica da madeira $\left(\mathrm{g} / \mathrm{cm}^{3}\right)$;

$M_{0}=$ massa da amostra na condição de $0 \%$ de umidade $(\mathrm{g})$;

$V_{0}=$ volume da amostra em condição saturada $\left(\mathrm{cm}^{3}\right)$;

$\Phi=$ porosidade da madeira $(\%)$.

\subsection{Eficiência dos retardantes de chama na madeira}

O processo de combustão da madeira foi realizado de acordo com metodologia descrita por TONDI et al. [14], a qual visa avaliar o percentual de perda de massa das amostras no tempo. Torna-se importante ressaltar que a perda de massa foi avaliada em percentual devido à diferença de massa que há entre cada amostra, o que ocorre devido a heterogeneidade da madeira e assim, a variação de densidade entre estas. Para o ensaio de combustão, as amostras foram fixadas sobre uma balança de precisão e submetidas a combustão de um bico de Bunsen abastecido com gás liquefeito de petróleo (GLP), até ocorrer a perda de $80 \%$ de sua massa. Durante este processo, a cada 30 segundos o peso da amostra indicado no leitor da balança foi registrado. Dessa forma, é possível verificar a capacidade protetora que o retardante de chamas exerce sobre a madeira. Durante o processo de combustão, as amostras não ficam em contato direto com a chama, permanecendo suspensas a uma distância de nove centímetros $(9 \mathrm{~cm})$ do queimador.

\section{RESULTADOS E DISCUSSÃO}

\subsection{Caracterização física das espécies estudadas}

$\mathrm{Na}$ Tabela 2, constam os valores médios de densidade aparente e percentual de porosidade das amostras do presente estudo. 
Tabela 2: Propriedades físicas das espécies de madeireiras estudadas.

\begin{tabular}{l|l|l|l}
\hline ESPÉCIE & $\begin{array}{l}\text { DENSIDADE BÁSICA } \\
\text { MÉDIA } \mathbf{( g / \mathbf { c m } ^ { 3 } )}\end{array}$ & $\begin{array}{l}\text { DENSIDADE APARENTE } \\
\text { MÉDIA }\left(\mathbf{g} / \mathbf{c m}^{3}\right)\end{array}$ & $\begin{array}{l}\text { POROSIDADE } \\
\text { MÉDIA (\%) }\end{array}$ \\
\hline Eucalyptus. grandis & 0,442 & 0,527 & 71,33 \\
Eucalyptus saligna & 0,742 & 0,875 & 51,80 \\
Corymbia maculata & 0,773 & 0,917 & 49,79 \\
Eucalyptus dunnii & 0,680 & 0,833 & 55,85 \\
\hline
\end{tabular}

Segundo MORESCHI [6], a densidade da madeira pode ser considerada um dos índices de qualidade mais importante desta, uma vez que se relaciona diretamente com a produção de massa seca e com suas propriedades físicas e mecânicas. CARNEIRO et. al [15], afirmam que o aumento na densidade da madeira possui relação direta com a idade da árvore, ou seja, árvores de lenho juvenil apresentam menores valores de densidade que árvores de lenho adulto e ainda, que há uma tendência de estabilização na densidade da madeira após determinada idade da planta. RIBEIRO e FILHO [16], em seu trabalho, constaram densidades básicas do Eucalyptus grandis, Eucalyptus dunnii e Eucalyptus saligna, todos com lenho juvenil variando de $0,374 \mathrm{~g} / \mathrm{cm}^{3}$ a $0,503 \mathrm{~g} / \mathrm{cm}^{3}, 0,459 \mathrm{~g} / \mathrm{cm}^{3}$ a $0,580 \mathrm{~g} / \mathrm{cm}^{3}$ e $0,360 \mathrm{~g} / \mathrm{cm}^{3}$ a $0,518 \mathrm{~g} / \mathrm{cm}^{3}$ respectivamente. STURION et. al [17] verificaram que Corymbia maculata apresenta madeira mais densa que Eucalyptus grandis e Eucalyptus saligna, algo que corrobora os dados obtidos no presente estudo.

Além disso, BURGER e RICHTER [18], alegam que "a densidade da madeira, no geral, pode variar de 0,13 a $1,40 \mathrm{~g} / \mathrm{cm}^{3}$ e que esta característica está diretamente relacionada ao volume de poros vazios". Diante do exposto e de acordo com RIBEIRO e FILHO [16], CARNEIRO et. al [15] e BURGER e RICHTER [18], os dados de densidade aparente, densidade básica e percentual de porosidade obtidos neste estudo, conforme tabela 2 , estão de acordo com as premissas destes autores.

\subsection{Produtos retardantes de chamas nas madeiras}

A capacidade dos retardantes em reduzir a perda de massa das amostras ao longo do tempo permitiu gerar curvas gravimétricas, conforme Figuras 2, 3 e 4. Na Figura 2 (A) foi possível avaliar o comportamento da madeira de Eucalyptus grandis tratada com os produtos retardantes de chamas do presente estudo, ao serem submetidas a elevadas temperaturas. A Figura 2 (B) avaliou a massa residual da espécie Eucalyptus saligna tratada com os retardantes de chamas ao, ser submetida à combustão. Já a Figura 3 (C) e Figura 3 (D) avaliou a massa residual média da Corymbia maculata e do Eucalyptus dunni, respectivamente, quando tratadas com os produtos retardantes de chamas aqui estudados. Na Figura 4 (E) foram realizadas comparações de percentual de massa residual no tempo entre as quatro espécies florestais do estudo quando tratadas com o produto retardante CNB. Por fim, a Figura 4 (F) permitiu avaliar a massa residual ao longo do tempo, entre as quatro espécies florestais, quando tratadas com RSPAI.
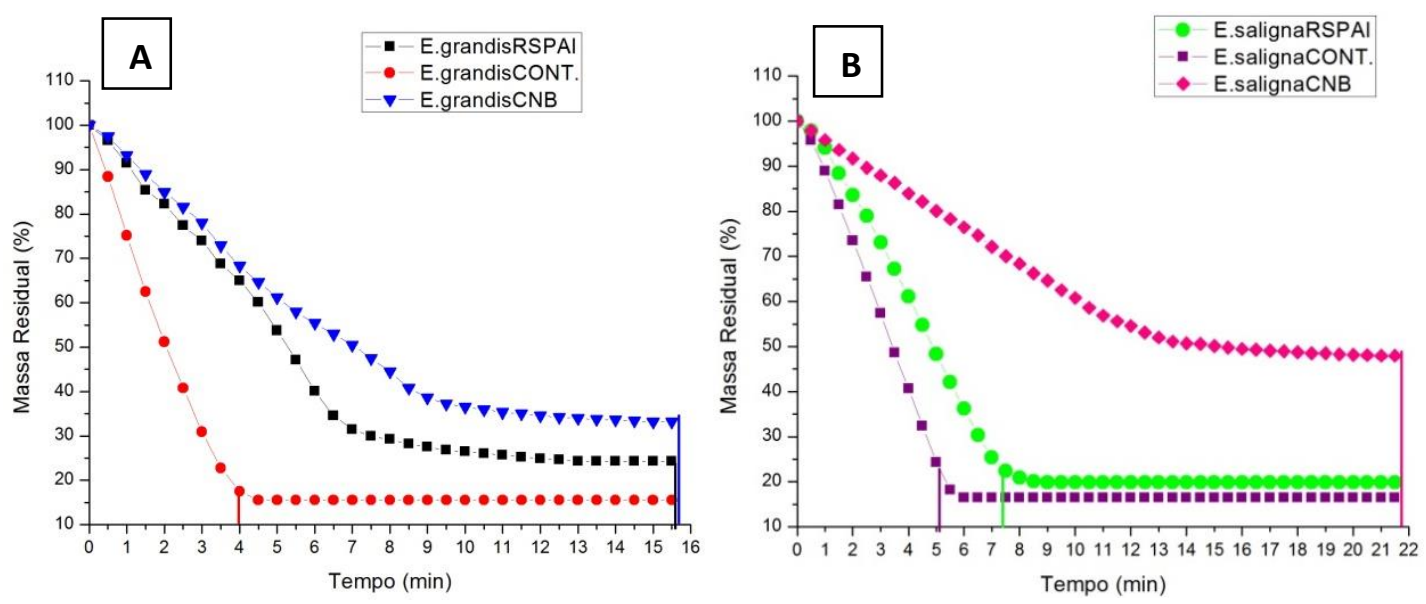

Figura 2: Massa residual do Eucalyptus grandis (A) e do Eucalyptus saligna (B) no tempo com diferentes tratamentos.

Utilizando o método da menor diferença significativa (LSD) de Fisher, com nível de confiança de 95\%, foi possível avaliar e evidenciar a importância dos tratamentos retardantes de chamas nas madeiras do 
estudo, relacionando a redução na perda de massa das amostras, conforme Tabelas 3, 4, 5 .

Para a avaliação da massa residual da madeira de Eucalyptus grandis, na Tabela 3, sob os tratamentos do presente estudo, quando submetidas a elevadas temperaturas, foi possível perceber que as amostras tratadas com tinta ignífuga (RSPAI) e com retardante de chamas à base de nitrogenados e boratos (CNB) não apresentaram diferenças significativas entre si com relação à massa residual no tempo, mas ambos apresentaram diferença na capacidade retardante de perda de massa das amostras quando comparado com o Eucalyptus grandis controle, indicando que esses dois tratamentos foram capazes de retardar a perda de massa desta espécie florestal.

Ainda na Tabela 3, foi possível observar que, os três tratamentos diferiram entre si quando aplicados no Eucalyptus saligna, e que o tratamento a base de nitrogenados e boratos (CNB) se mostrou mais eficiente dentre todos os tratamentos, visto que o mesmo impediu que o Eucalyptus saligna perdesse $80 \%$ de sua massa durante a combustão (Figura 2-B). Isso está relacionado ao fato desse retardante de chamas possuir consistência líquida e ser mais fluido que a tinta ignífuga, a qual se apresenta com textura mais viscosa. De acordo com TONDI et al. [14] quando a viscosidade da solução aumenta, a penetração na madeira não ocorre de forma homogênea. Portanto, um retardante mais fluido possui capacidade de penetração mais homogênea e mais profunda nas amostras, gerando assim, uma proteção mais eficaz.

Tabela 3: Análise estatística de massa média das amostras de Eucalyptus grandis e Eucalyptus saligna sob os diferentes tratamentos retardantes de chamas.

\begin{tabular}{ll|l|l|l|l|l|l}
\hline & \multicolumn{6}{c}{ TRATAMENTOS } \\
\cline { 2 - 7 } & \multicolumn{3}{c}{$\begin{array}{c}\text { Eucalyptus grandis } \\
\text { CONT }\end{array}$} & \multicolumn{3}{|c}{ Eucalyptus saligna } \\
MASSA RESIDUAL MÉDIA NO TEMPO (\%) & $17,47 \mathrm{a}$ & $64,96 \mathrm{~b}$ & $68,35 \mathrm{~b}$ & $18,25 \mathrm{a}$ & $42,14 \mathrm{~b}$ & $78,27 \mathrm{c}$ \\
DESVIO PADRÃO & 1,29 & 8,00 & 7,31 & 3,69 & 14,38 & 1,92 \\
COEFICIENTE DE VARIAÇÃO (\%) & 7,41 & 12,31 & 10,69 & 20,23 & 34,12 & 2,45 \\
\hline VALOR DE F & 61,18 & & 36,69 & \\
\hline
\end{tabular}

Médias seguidas pela mesma letra na linha, não diferem estatisticamente entre si, de acordo com o método da menor diferença significativa (LSD) de Fisher, com nível de confiança de 95\%.
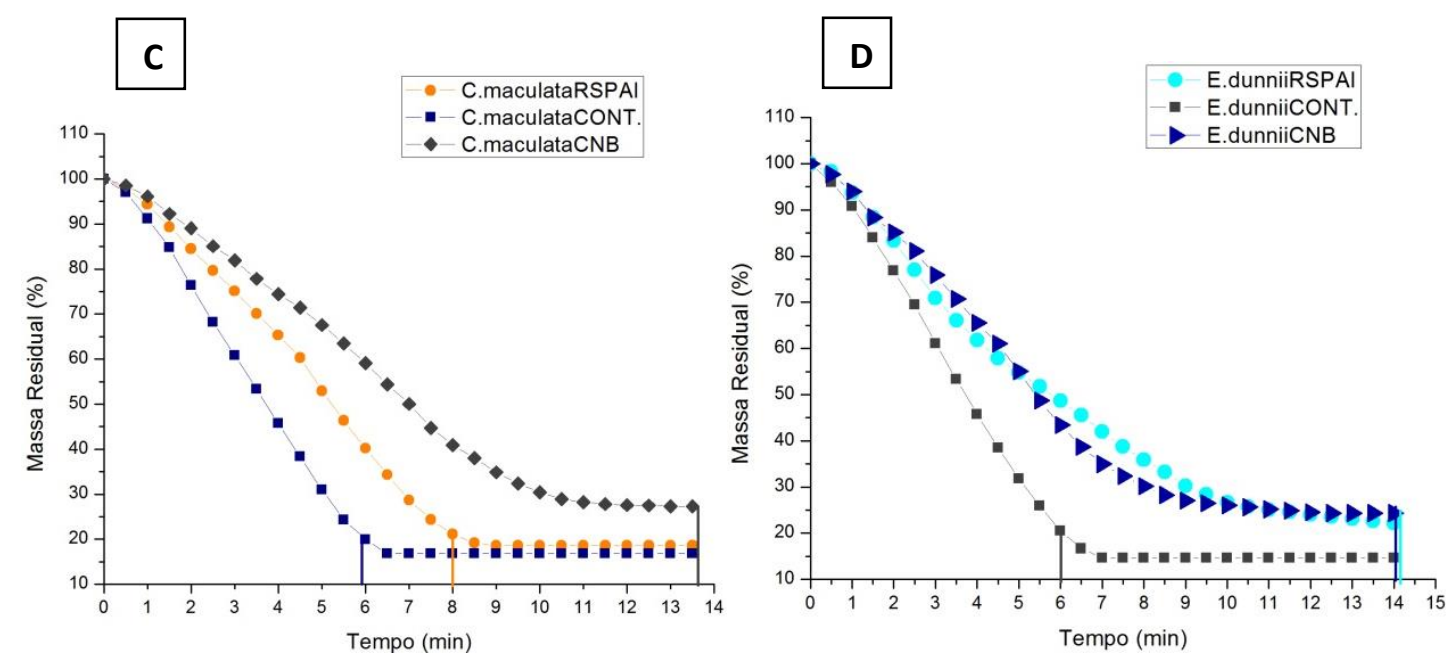

Figura 3: Massa residual da Corymbia maculata (C) e do Eucalyptus dunnii (D) no tempo com diferentes tratamentos.

Com relação a perda de massa da madeira de Corymbia maculata (Tabela 4) sob os diferentes tratamentos retardantes de chamas quando em elevadas temperaturas, verificou-se que as amostras tratadas não diferem significativas entre si, mas ambos apresentam capacidade retardante na perda de massa quando comparado ao controle, indicando que estes tratamentos apresentam eficácia no retardo na perda de massa e ainda, que o retardante a base de nitrogenados e boratos (CNB) (Figura $3 \mathrm{C}$ ), dentre os dois tratamentos, mostra-se mais eficiente. 
Na Tabela 4 ainda, foi possível analisar que no Eucalyptus dunnii tratado com os retardantes de chamas do presente estudo, não apresentou diferenças significativas entre si, mas ambos também indicaram ter capacidade retardante na perda de massa quando comparado com o tratamento controle. De acordo com TONDI et al. [14], tal comportamento, tanto na Corymbia maculata quando no Eucalyptus dunnii ocorreu por que o boro já está incluído na formulação natural da madeira devido ao seu alto desempenho com propriedades antibiológicas, e portanto, assim ocorre um efeito duplamente benéfico da presença deste componente neste polímero.

Tabela 4: Análise estatística de massa residual média das amostras de Corymbia maculata e Eucalyptus dunnii sob os diferentes tratamentos retardantes de chamas.

\begin{tabular}{|c|c|c|c|c|c|c|}
\hline & \multicolumn{6}{|c|}{ TRATAMENTOS } \\
\hline & \multicolumn{3}{|c|}{ Corymbia maculata } & \multicolumn{3}{|c|}{ Eucalyptus dunnii } \\
\hline & CONT & RSPAI & CNB & CONT & RSPAI & CNB \\
\hline MASSA RESIDUAL MÉDIA NO TEMPO (\%) & $19,97 \mathrm{a}$ & $40,24 \mathrm{ab}$ & $59,09 \mathrm{~b}$ & $16,64 \mathrm{a}$ & $45,57 \mathrm{~b}$ & $38,68 b$ \\
\hline DESVIO PADRÃO & 3,48 & 13,5 & 14,18 & 2,89 & 10,12 & 7,17 \\
\hline COEFICIENTE DE VARIAÇÃO (\%) & 17,41 & 33,56 & 24,01 & 17,39 & 22,2 & 18,53 \\
\hline VALOR DE F & \multicolumn{3}{|l|}{8,71} & \multicolumn{3}{|l|}{12,68} \\
\hline
\end{tabular}
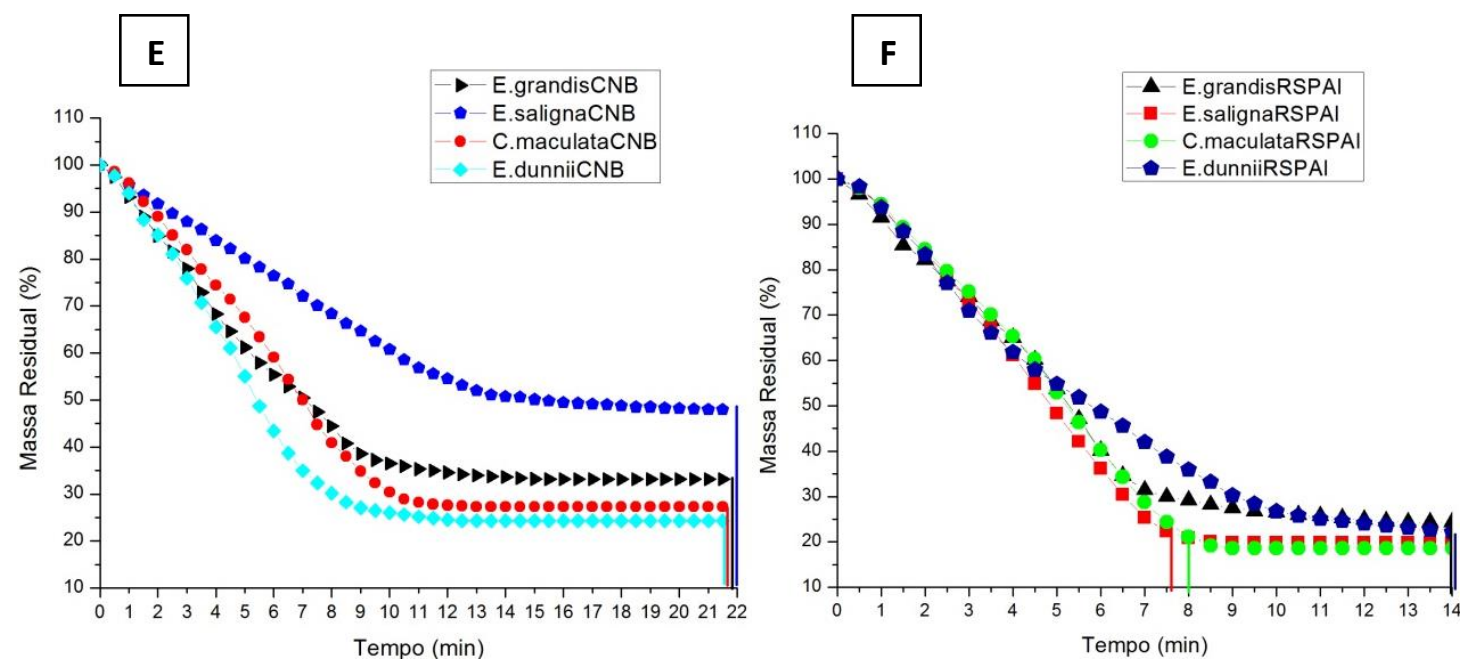

Figura 4: Massa residual média das amostras tratadas com retardante de chamas CNB (E) e com RSPAI (F).

Ao avaliar a capacidade retardante na perda de massa das madeiras de eucalipto no presente estudo, quando tratadas com o retardante de chamas a base de nitrogenados e boratos (CNB) (Tabela 5), foi possível perceber que Eucalyptus saligna foi a espécie que apresentou menor perda de massa no tempo em relação as demais. Já, quando as mesmas análises foram feitas, mas agora com o retardante a base de resina sintética com aditivos ignífugos (RSPAI), verificou-se que Eucalyptus dunnii se diferiu significativamente das demais espécies aqui estudadas. Isso possivelmente ocorreu devido a reação e interação química entre os compostos nitrogenados e boratos presentes no retardante avaliado, e a heterogeneidade na composição química das referidas espécies de eucalipto, conforme indicado por PAULA et al. [11], onde alega que compostos de boro e fósforo, atuam absorvendo o calor ambiente, evitando portanto, a formação de gases inflamáveis durante a combustão, consequentemente, evitando a pirólise do material. Já o retardante a base de resina sintética pigmentada com aditivos ignífugos (RSPAI), de acordo com ABARCA [12], atua apenas como uma camada protetora sobre a madeira, a qual impede a formação e eliminação de gases tóxicos e inflamáveis que alimentariam a combustão da madeira. 
Tabela 5: Análise estatística de percentual de massa residual média das amostras tratadas com CNB e amostras tratadas com RSPAI.

\begin{tabular}{l|l|l}
\hline \multicolumn{2}{c}{ MASSA RESIDUAL MÉDIA NO TEMPO $(\%)$} \\
\hline AMOSTRAS DE MADEIRAS & AMOSTRAS TRATADAS COM CNB & \multicolumn{1}{c}{ AMOSTRAS TRATADAS COM RSPAI } \\
\hline \multirow{2}{*}{$\begin{array}{l}\text { Eucalyptus dunnii } \\
\text { Corymbia maculata }\end{array}$} & $24,32 \mathrm{a} *(6,36)(26,14)$ & $33,19 \mathrm{~b} *(9,79)(29,50)$ \\
\cline { 2 - 3 } $\begin{array}{l}\text { Eucalyptus grandis } \\
\text { Eucalyptus saligna }\end{array}$ & $27,29 \mathrm{ab} *(1,36)(4,98)$ & $19,19 \mathrm{a} *(0,62)(3,25)$ \\
\cline { 2 - 3 } & $33,96 \mathrm{~b} *(4,47)(15,16)$ & $28,20 \mathrm{ab} *(6,56)(23,26)$ \\
\cline { 2 - 3 } & $52,01 \mathrm{c} *(1,13)(2,17)$ & $20,08^{\mathrm{a}} *(0,47)(2,32)$ \\
\cline { 2 - 3 } & Valor $\mathrm{F}=29,11$ & Valor $\mathrm{F}=3,88$ \\
\hline
\end{tabular}

Com base nos resultados encontrados, é possível afirmar que os tratamentos, quando comparados com as amostras controle, apresentaram resultados satisfatórios no que diz respeito a sua capacidade de retardo à perda de massa no tempo das espécies de eucalipto do presente estudo submetidas a elevadas temperaturas, algo observado por TONDI et al. [14], onde o autor afirma que o uso do boro na formulação retarda o tempo de brasa e a perda de peso significativa da madeira quando em combustão.

\section{CONCLUSÃO}

Com base nos resultados apresentados e discutidos em relação à capacidade retardante de perda de massa que os diferentes retardantes comerciais conferem às madeiras de eucalipto (Eucalyptus grandis, Eucalyptus saligna, Corymbia maculata e Eucalyptos dunnii), pode ser possível afirmar que:

- Ambos retardantes de chamas mostram-se eficazes no que diz respeito a capacidade retardante de perda de massa das amostras de Eucaliptos quando em elevadas temperaturas.

- O retardante de chamas a base de nitrogenados e boratos se mostra mais eficiente em relação ao retardante de chamas a base de resina sintética pigmentada com aditivos ignífugos (tinta ignífuga).

- Com relação a eficácia dos retardantes de chamas sobre as espécies de Eucaliptos, os resultados variam de acordo com a espécie estudada e isso ocorre possivelmente devido a composição química de cada madeira deste estudo.

\section{AGRADECIMENTOS}

A CMPC Celulose pela doação das amostras que permitiram a realização do presente estudo e a CAPES, por fomentar os estudos desenvolvidos por esta equipe de trabalho.

\section{BIBLIOGRAFIA}

[1] FIGUEROA, M. J. M., MORAES, P. D. “Comportamento da madeira a temperaturas elevadas". Revista Ambiente Construído, v. 9, n. 4, pp. 157-174, Porto Alegre, Dez. 2009.

[2] QUIRINO, W. F., VALE, A. T. ANDRADE, A. P. A., et al., "Poder calorífico da madeira e de materiais ligno-celulósicos", Revista da Madeira, n.89, pp.100-106, 2005.

[3] TRUGILHO, P. F., LIMA, J. T., MENDES, L. M. "Influência da idade nas características físico-químicas e anatômicas da madeira de Eucalyptus saligna", Revista Cerne, v. 2, n. 1, pp. 94-11, 1996.

[4] PEDIEU R., KOUBAA, A., RIEDL, B., et al., "Fire-retardant properties of wood particleboards treated with boric acid". European Journal of Wood and Wool Products. 2011.

[5] CASTRO, A. Uma investigação teórico-experimental da combustão de madeira, Dissertação M.Sc., INPE, São José dos Campos, SP, Brasil, 2005.

[6] MORESCHI, J. C., Propriedades da madeira. $5^{\mathrm{a}}$ ed. Departamento de Engenharia e Tecnologia Florestal da UFPR. Curitiba/PR, 2005.

[7] AGUILlAR, D. F. Combustibilidade e tratamento ignífugo da madeira, Dissertação de M. Sc., Departamento de Arquitetura, USP-EESC, São Carlos/ SP, 1986.

[8] RIBEIRO, G. A., LIMA, G. S., OLIVEIRA, A. L. S., et al., "Eficiência de um retardante de longa duração na redução da propagação do fogo", Revista Árvore, v. 30, n. 6 pp. 1025-1031. Novembro, 2006.

[9] FURTAT, P. P., Processamento e caracterização de blendas de poliestireno com retardância à chama. Dissertação de M. Sc., UFSC, Florianópolis/ SC., 2014. 
[10] WILKIE, C. A., MORGAN, A. B. (Eds.). Fire Retardancy of Polymeric Materials. $2^{\mathrm{a}}$ ed. CRC Press, 2009.

[11] PAULA, M. O., MOREIRA, G. R., SEGUNDINHO. “Análise termomecânica da madeira de Corymbia citriodora submetida a gradientes térmicos”, Floresta e Ambiente, v. 21, n.3, pp.358-368, Julho, 2014.

[12] ABARCA, S. A. C. Obtenção de poliestireno expansível com retardância chama via produção in situ, Dissertação de M. Sc. UFSC, Florianópolis/SC, 2010.

[13] Remade, REVISTA DA MADEIRA, edição 59, http://www.remade.com.br/. Acessado em setembro de 2017.

[14] TONDI, G., WIELAND, S., WIMMER T. et al.; "Tannin-boron preservatives for wood buildings: mechanical and fire properties", European Journal of Wood and Wood Products, v. 70, n 5, pp 689-696. Sep. 2012.

[15] CARNEIRO, A. C. O., CASTRO, A. F. N. M., CASTRO , O. R. V. et al., "Potencial energético da madeira de Eucalyptus sp. em função da idade e de diferentes materiais genéticos", Revista Árvore, v.38, n.2, pp.375-381. Viçosa/ MG. 2014.

[16] RIBEIRO, F. A., FILHO, J. Z. "Variação da densidade básica da madeira em espécies/procedências de Eucalyptos spp", Instituto de Pesquisas e Estudos Florestais (IPEF), n. 46. pp. 76-85, Araraquara/ SP, 2003.

[17] STURION, J. A. PEREIRA, J. C. D., ALBINO, J. C. et al. "Variação da densidade básica da madeira de doze espécies de Eucalyptus plantadas em Uberlândia, Minas Gerais”, In Boletim de pesquisa florestal, Colombo/ PR. pp. 28-38, Junho 1987.

[18] BURGER, L. M.; RICHTER, H. G. Anatomia da madeira. São Paulo/SP, NOBEL, 1991.

[19] ASSOCIAÇÃO BRASILEIRA DE NORMAS TÉCNICAS - ABNT. NBR 7190: projeto de estruturas de madeira. Ensaios de caracterização. Rio de Janeiro/ RJ. 1997. 107 p. 